Article

\title{
Evolution Inclusions in Banach Spaces under Dissipative Conditions
}

\author{
Tzanko Donchev ${ }^{1, *}$, Shamas Bilal ${ }^{2}$, Ovidiu Cârjă ${ }^{3,4}$, Nasir Javaid ${ }^{5}$ and Alina I. Lazu ${ }^{6}$ \\ 1 Department of Mathematics, University of Architecture, Civil Engineering and Geodesy, Sofia 1164, Bulgaria \\ 2 Department of Mathematics, University of Sialkot, Sialkot 51040, Pakistan; Shams.bilal@uskt.edu.pk \\ 3 Department of Mathematics, “Al. I. Cuza” University, Iaşi 700506, Romania; ocarja@uaic.ro \\ "Octav Mayer" Mathematics Institute, Romanian Academy, Iaşi 700505, Romania \\ Abdus Salam School of Mathematical Sciences, Lahore 54000, Pakistan; nasir.jav7000@gmail.com \\ 6 Department of Mathematics, “Gh. Asachi” Technical University, Iaşi 700506, Romania; \\ vieru_alina@yahoo.com \\ * Correspondence: tzankodd@gmail.com
}

Received: 23 March 2020; Accepted: 5 May 2020; Published: 9 May 2020

check for updates

\begin{abstract}
We develop a new concept of a solution, called the limit solution, to fully nonlinear differential inclusions in Banach spaces. That enables us to study such kind of inclusions under relatively weak conditions. Namely we prove the existence of this type of solutions and some qualitative properties, replacing the commonly used compact or Lipschitz conditions by a dissipative one, i.e., one-sided Perron condition. Under some natural assumptions we prove that the set of limit solutions is the closure of the set of integral solutions.
\end{abstract}

Keywords: m-dissipative operators; limit solutions; integral solutions; one-sided Perron condition; Banach spaces

\section{Introduction and Preliminaries}

Let $X$ be a real Banach space with the norm $|\cdot|, A: D(A) \subset X \rightrightarrows X$ an $\mathrm{m}$-dissipative operator generating the semigroup $\{S(t): \overline{D(A)} \rightarrow \overline{D(A)} ; t \geq 0\}$ and $F: I \times X \rightrightarrows X$ a multifunction with nonempty, closed and bounded values, where $I=\left[t_{0}, T\right]$.

In this paper, we study evolution inclusions of the form

$$
\dot{x}(t) \in A x(t)+F(t, x(t)), x\left(t_{0}\right)=x_{0} \in \overline{D(A)} .
$$

Notice that many parabolic systems can be written in the form (1). We refer the reader to [1-3] for the general theory of the system (1) when $F$ is single valued. In the case when $X^{*}$ is uniformly convex, the system (1) is comprehensively studied in [4]. We recall also the monograph [5], where (1) is studied in different settings.

An important problem regarding the system (1) is to find the closure of the set of integral solutions. This problem is not solved in the case of general Banach spaces.

We consider the associated Cauchy problem

$$
\dot{x}(t) \in A x(t)+f(t), x\left(t_{0}\right)=x_{0} \in \overline{D(A)},
$$

where $f(\cdot)$ is a Bochner integrable function. We denote by $[\cdot, \cdot]_{+}$the right directional derivative of the norm, i.e., $[x, y]_{+}=\lim _{h \rightarrow 0^{+}} h^{-1}(|x+h y|-|x|)$ (see, e.g., ([6], Section 1.2) for definition and properties). 
Following [7], we say that a continuous function $x:\left[t_{0}, T\right] \rightarrow \overline{D(A)}$ is an integral solution of (2) on $\left[t_{0}, T\right]$ if $x\left(t_{0}\right)=x_{0}$ and for every $u \in D(A), v \in A u$ and $t_{0} \leq \tau<t \leq T$ the following inequality holds

$$
|x(t)-u| \leq|x(\tau)-u|+\int_{\tau}^{t}[x(s)-u, f(s)+v]_{+} d s
$$

Definition 1. The Bochner integrable function $g(\cdot)$ is said to be pseudoderivative of the continuous function $y(\cdot)$ (with respect to $A$ ) if $y(\cdot)$ is an integral solution of $(2)$ on $\left[t_{0}, T\right]$ with $f(\cdot)$ replaced by $g(\cdot)$.

Notice that the pseudoderivative $g(\cdot)$ (if it exists) depends on $A$ and $y(\cdot)$. However, along this paper $A$ is fixed and we assume without loss of generality that the pseudoderivative depends only on $y(\cdot)$. To stress this dependence on $y$, we will denote the pseudoderivative $g(\cdot)$ by $g_{y}(\cdot)$.

It is well known that for each $x_{0} \in \overline{D(A)}$ the Cauchy problem (2) has a unique integral solution on $\left[t_{0}, T\right]$. Moreover, if $x(\cdot)$ and $y(\cdot)$ are integral solutions of (2) with $x\left(t_{0}\right)=x_{0}$ and $y\left(t_{0}\right)=y_{0}$ then

$$
\begin{gathered}
|x(t)-y(t)| \leq\left|x_{0}-y_{0}\right|+\int_{t_{0}}^{t}\left[x(s)-y(s), f_{x}(s)-f_{y}(s)\right]_{+} d s, \\
|x(t)-y(t)| \leq\left|x_{0}-y_{0}\right|+\int_{t_{0}}^{t}\left|f_{x}(s)-f_{y}(s)\right| d s,
\end{gathered}
$$

for every $t \in\left[t_{0}, T\right]$ (see, e.g., [7]).

We define now the notion of integral solution for the differential inclusion (1). Moreover, following [8], where the semilinear case was considered, we define the notions of $\varepsilon$-solution (called outer $\varepsilon$-solution in [8]) and limit solution for (1). In the following, $\mathbb{B}$ denotes the closed unit ball in $X$.

Definition 2. The function $x: I \rightarrow \overline{D(A)}$ is said to be an integral solution of (1) on I if it is an integral solution of (2) such that its pseudoderivative $f_{x}(\cdot)$ satisfies $f_{x}(t) \in F(t, x(t))$ for a.a. $t \in I$.

Consider the following system

$$
\left\{\begin{array}{l}
\dot{x}(t) \in A x(t)+F(t, x(t)+\mathbb{B})+\mathbb{B}, \\
x\left(t_{0}\right)=x_{0} .
\end{array}\right.
$$

Definition 3. (i) Let $\varepsilon>0$. The continuous function $x: I \rightarrow \overline{D(A)}$ is said to be an $\varepsilon$-solution of (1) on I if it is a solution of (5) and its pseudoderivative $f_{x}(\cdot)$ satisfies

$$
\int_{I} \operatorname{dist}\left(f_{x}(t), F(t, x(t))\right) d t \leq \varepsilon .
$$

(ii) The function $x(\cdot)$ is said to be a limit solution of (1) on I if $x(t)=\lim _{n \rightarrow \infty} x_{n}(t)$ uniformly on I for some sequence $\left(x_{n}(\cdot)\right)$ of $\varepsilon_{n}$-solutions as $\varepsilon_{n} \downarrow 0^{+}$.

Recall that the distance between a point $u \in X$ and a subset $C$ of $X$ is given by $\operatorname{dist}(u ; C)=$ $\inf \{\|u-c\| ; c \in C\}$.

In the literature, we can find different definitions for $\varepsilon$-solutions. Maybe the most popular is when its pseudoderivative satisfies $f_{x}(t) \in F(t, x(t)+\varepsilon \mathbb{B})$ a.e. on $I$. However, our definition given above is more convenient for the study of the qualitative properties of the set of integral solutions of (1) in the case when $X$ is an arbitrary Banach space.

For ordinary differential inclusions $(A=0)$, the limit solutions are usually called quasitrajectories (cf., [9] ). We prefer the notion of limit solution because it is the original definition of the integral solution in the case of m-dissipative systems (cf. [6]). For ordinary differential inclusions in $\mathbb{R}^{n}$, 
the limit solutions are the integral solutions of the relaxed system. In our case, the relaxed system has the form

$$
\dot{x}(t) \in A x(t)+\overline{c o} F(t, x(t)), x\left(t_{0}\right)=x_{0},
$$

where $\overline{c o} F(t, x(t))$ stands for the closed convex hull of the set $F(t, x(t))$. In this general setting, the limit solutions are not integral solutions of the relaxed system (6).

It is well known that the set of integral solutions of (6) is not necessarily closed in $C(I, X)$ even if $X$ is finite dimensional. For instance, in [10] the author constructed an example in which a sequence $\left(x_{n}(\cdot)\right)$ of integral solutions of

$$
\dot{x}(t) \in A x(t)+f_{n}(t), x\left(t_{0}\right)=x_{0},
$$

converges uniformly on $\left[t_{0}, T\right]$ to a function $x(\cdot),\left(f_{n}(\cdot)\right)$ converges weakly in $L^{1}\left(t_{0}, T ; X\right)$ to $f(\cdot)$, but $x(\cdot)$ is not an integral solution of

$$
x^{\prime}(t) \in A x(t)+f(t), x\left(t_{0}\right)=x_{0} .
$$

The main results of this paper are summarized as follows.

(I) We prove that the set of limit solutions of (1) is nonempty and closed in $C(I, X)$ when $X$ is a general Banach space and $F(\cdot, \cdot)$ is almost continuous and satisfies a one-sided Perron condition.

(II) We prove that in the case when $A$ generates a compact semigroup, the closure of the set of integral solutions of (1) is exactly the set of limit solutions, which in general does not coincide with the set of integral solutions of the relaxed system. The same result is proved also when $F(t, \cdot)$ is full Perron, but without any restrictions on the semigroup $A$.

The limit solutions in the case when $A$ is linear were studied in [8]. It was shown there that the limit solutions of (1) and (6) coincide. It is not the case for the nonlinear problem.

Let us now define a few classes of multifunctions which will be used in the following.

We say that $F(\cdot, \cdot)$ is lower semicontinuous (LSC) at $\left(t_{0}, x_{0}\right) \in I \times X$ if for every $f_{0} \in F\left(t_{0}, x_{0}\right)$, every $x_{k} \rightarrow x_{0}$ and every $t_{k} \rightarrow t_{0}$ there exists $f_{k} \in F\left(t_{k}, x_{k}\right)$ such that $f_{k} \rightarrow f_{0}$. This definition is equivalent to the following property of the graph: for every $\alpha \in F\left(t_{0}, x_{0}\right)$ and every $\varepsilon>0$, there exists $\delta>0$ such that $\alpha \in F(t, x)+\varepsilon \mathbb{B}$, when $\left|t-t_{0}\right| \leq \delta$ and $\left|x-x_{0}\right| \leq \delta$.

The multifunction $F(\cdot, \cdot)$ is called LSC if it is LSC at every $(t, x) \in I \times X$.

The multifunction $F(\cdot, \cdot)$ is called continuous if it is continuous with respect to the Hausdorff distance. We recall that the Hausdorff distance between the bounded sets $B$ and $C$ is defined by

$$
D_{H}(B ; C)=\max \{e(B ; C), e(C ; B)\},
$$

where $e(B ; C)$ is the excess of $B$ to $C$, defined by $e(B ; C)=\sup _{x \in B} \operatorname{dist}(x ; C)$.

The multifunction $F(\cdot, \cdot)$ is called almost LSC (continuous) if for every $\varepsilon>0$ there exists a compact set $I_{\varepsilon} \subset I$ with Lebesgue measure meas $\left(I \backslash I_{\varepsilon}\right) \leq \varepsilon$ such that $\left.F\right|_{I_{\varepsilon} \times X}$ is LSC (continuous).

Let $v: I \times \mathbb{R}_{+} \rightarrow \mathbb{R}_{+}$be Carathéodory and integrally bounded on the bounded sets. As is well known, the scalar differential equation

$$
\dot{r}(t)=v(t, r(t)), \quad r\left(t_{0}\right)=r_{0} \geq 0,
$$

has maximal solutions $h(\cdot)$, i.e., $0 \leq r(t) \leq h(t)$ for every solution $r(\cdot)$ of $(7)$ on the existence interval of $h(\cdot)$ (see, e.g., [6]).

We introduce now the standing hypotheses of this paper.

Hypothesis $\mathbf{1}$ (H1). The multifunction $F(\cdot, \cdot)$ is almost continuous. 
Hypothesis 2 (H2). There exists $\gamma>0$ such that $\|F(t, x)\| \leq \gamma(1+|x|)$ for a.a. $t \in I$ and every $x \in X$. We recall that $\|F(t, x)\|=\sup _{y \in F(t, x)}|y|$.

Hypothesis 3 (H3). (One-sided Perron condition) There exist a Perron function $w(\cdot, \cdot)$ and a null set $\mathcal{N} \subset I$ such that such for every $x, y \in X$, for every $\varepsilon>0$ and for every $f \in F(t, x)$ there exists $g \in F(t, y)$ such that

$$
[x-y, f-g]_{+} \leq w(t,|x-y|)+\varepsilon
$$

on $I \backslash \mathcal{N}$.

We recall that the Carathéodory function $w: I \times \mathbb{R}_{+} \rightarrow \mathbb{R}_{+}$is said to be Perron function if it is integrally bounded on bounded sets, $w(t, 0) \equiv 0, w(t, \cdot)$ is nondecreasing for every $t \in I$ and the zero function is the only solution of the scalar differential equation $r^{\prime}(t)=w(t, r(t)), r\left(t_{0}\right)=0$, on $I$.

Notice that it is more popular to call such kind of functions Kamke functions. We refer the reader to [11], where Perron and Kamke functions are comprehensively studied. That paper is the main reason to use here the notion of Perron (not Kamke) function. In [12] some examples of the Perron (Kamke) functions different from the Lipshitz one are given (see, e.g., Corollary 1.13 and Corollary 1.15).

Remark 1. Due to Gronwall's lemma, there exists a constant $M>0$ such that $|x(t)| \leq M$ for every $t \in I$ and every solution $x(\cdot)$ of $(5)$. Let $N=1+\gamma(2+M)$. Then $\|F(t, x(t)+\mathbb{B})+\mathbb{B}\| \leq N$ for every solution $x(\cdot)$ of (5).

Clearly, for every solution $x(\cdot)$ of (5), in particular for every $\varepsilon$-solution $x(\cdot)$ of (1), with the pseudoderivative $f_{x}(\cdot)$, we have that $\operatorname{dist}\left(f_{x}(t), F(t, x(t))\right) \leq 2 N$ on $I$, since $\left|f_{x}(t)\right| \leq N$ and $\|F(t, x(t))\| \leq N$ for every $t \in I$.

\section{Main Results}

The main results are given in three subsections. In the first one, we prove the existence of limit solutions. In the second subsection, we prove the most interesting results of this paper, namely, that the set of limit solutions of (1) is the closure of the set of integral solutions of (1) when $A$ generates a compact semigroup or when $F(t, \cdot)$ is full Perron. An example and some applications are discussed in the last two subsections.

\subsection{Existence of Limit Solutions}

In this subsection we prove an existence result of $\varepsilon$-solutions of the Cauchy problem (1) on $I$ and a variant of the well known lemma of Filippov-Pliś.

First, recall that $\bar{t}$ is said to be a right dense point of a closed subset $\mathcal{I} \subset I$ if for every $\tau>0$ there exists a point $s \in(\bar{t}, \bar{t}+\tau) \cap \mathcal{I}$. Clearly, $\bar{t}$ is not a right dense point of $\mathcal{I}$ if there exists $\tau>0$ such that $(\bar{t}, \bar{t}+\tau) \bigcap \mathcal{I}=\varnothing$.

Lemma 1. Assume that $F(\cdot, \cdot)$ is almost LSC and satisfies (H2). Then for every $\varepsilon>0$ there exists at least one $\varepsilon$-solution of (1) defined on the whole I.

Proof. Let $\varepsilon>0$. We take $\varepsilon^{\prime} \leq \frac{\varepsilon}{T-t_{0}+2 N}$. There exists $I^{\prime} \subset I$ a closed set with Lebesgue measure meas $\left(I^{\prime}\right) \geq T-t_{0}-\varepsilon^{\prime}$ such that $\left.F\right|_{I^{\prime} \times X}$ is LSC on $I^{\prime} \times X$.

We take $f_{0} \in F\left(t_{0}, x_{0}\right)$ arbitrary but fixed and let $f_{1}(\cdot)$ be Bochner integrable with $f_{1}(t) \in F\left(t, x_{0}\right)$ on $I$. Two cases are possible. 
Case 1. If $t_{0}$ is a right dense point of $I^{\prime}$. Since $\left.F\right|_{I^{\prime} \times X}$ is LSC at $\left(t_{0}, x_{0}\right)$, then there exists $\delta \in(0,1 / 2)$ such that if $t \in I^{\prime}$ with $t-t_{0} \leq \delta$ and $\left|y-x_{0}\right| \leq \delta$ then $f_{0} \in F(t, y)+\varepsilon^{\prime} \mathbb{B}$. We pick

$$
f_{y}(t)=\left\{\begin{array}{l}
f_{0}, t \in I^{\prime} \\
f_{1}(t), t \in I \backslash I^{\prime}
\end{array}\right.
$$

Let $y_{1}(\cdot)$ be the integral solution of the Cauchy problem

$$
\dot{y}(t) \in A y(t)+f_{y}(t), y\left(t_{0}\right)=x_{0} .
$$

Since $\lim _{t \downarrow t_{0}} y_{1}(t)=x_{0}$, we deduce that there exists $\tau \in\left(t_{0}, t_{0}+\delta\right)$ such that $\left|y_{1}(t)-x_{0}\right| \leq \delta$ whenever $t \in\left[t_{0}, \tau\right)$. Thus, $f_{0} \in F\left(t, y_{1}(t)\right)+\varepsilon^{\prime} \mathbb{B}$ for every $t \in\left[t_{0}, \tau\right) \bigcap I^{\prime}$ and $f_{1}(t) \in F\left(t, y_{1}(t)+\mathbb{B}\right)$ for $t \in\left[t_{0}, \tau\right) \cap\left(I \backslash I^{\prime}\right)$. Therefore, $f_{y}(t) \in F\left(t, y_{1}(t)+\mathbb{B}\right)+\mathbb{B}$ for every $t \in\left[t_{0}, \tau\right)$, i.e., $y_{1}(\cdot)$ is a solution of $(5)$ on $\left[t_{0}, \tau\right)$.

We let $y(t)=y_{1}(t)$ for every $t \in\left[t_{0}, \tau\right)$. Thus, $\operatorname{dist}\left(f_{y}(t), F(t, y(t))\right) \leq \varepsilon^{\prime}$ for every $t \in\left[t_{0}, \tau\right) \bigcap I^{\prime}$ and, due to Remark 1 , $\operatorname{dist}\left(f_{y}(t), F(t, y(t))\right) \leq 2 N$ for every $t \in\left[t_{0}, \tau\right) \bigcap\left(I \backslash I^{\prime}\right)$.

Case 2. If $t_{0}$ is not a right dense point of $I^{\prime}$, let $y_{1}(\cdot)$ be the integral solution of the Cauchy problem

$$
\dot{y}(t) \in A y(t)+f_{1}(t), y\left(t_{0}\right)=x_{0} \text {. }
$$

Then there exists $\tau>t_{0}$ such that $\left[t_{0}, \tau\right) \subset I \backslash I^{\prime}$ and $\left|y_{1}(t)-x_{0}\right|<\varepsilon^{\prime}$ for $t \in\left[t_{0}, \tau\right)$. Thus, $y_{1}(\cdot)$ is a solution of (5) on $\left[t_{0}, \tau\right)$.

We let $y(t)=y_{1}(t)$ and $f_{y}(t)=f_{1}(t)$ for every $t \in\left[t_{0}, \tau\right)$. Moreover, $\operatorname{dist}\left(f_{y}(t), F(t, y(t))\right) \leq 2 N$ for every $t \in\left[t_{0}, \tau\right)$.

In both cases we let $y_{\tau}=\lim _{t \uparrow \tau} y(t)$. We continue the above construction in a similar way by replacing $t_{0}$ by $\tau$ and $x_{0}$ by $y_{\tau}$.

Let $\left[t_{0}, \bar{t}\right)$ be the maximal interval of the existence of solution $y(\cdot)$ of (5), with the properties that $\operatorname{dist}\left(f_{y}(t), F(t, y(t))\right) \leq \varepsilon^{\prime}$ on $\left[t_{0}, \bar{t}\right) \cap I^{\prime}$ and $\operatorname{dist}\left(f_{y}(t), F(t, y(t))\right) \leq 2 N$ on $\left[t_{0}, \bar{t}\right) \cap\left(I \backslash I^{\prime}\right)$, where $f_{y}(\cdot)$ is the pseudoderivative of $y(\cdot)$. Suppose that $\bar{t}<T$. Due to the growth condition $\lim _{t \uparrow \bar{t}} y(t)$ exists. Let $y_{\bar{t}}=\lim _{t \uparrow \bar{t}} y(t)$. Then, using a similar construction as above with $\bar{t}$ instead of $t_{0}$ and $y_{\bar{t}}$ instead of $x_{0}$, we can extend the solution $y(\cdot)$ on some interval $\left[t_{0}, \bar{t}+\theta\right), \theta>0$, such that $\operatorname{dist}\left(f_{y}(t), F(t, y(t))\right) \leq \varepsilon^{\prime}$ on $\left[t_{0}, \bar{t}+\theta\right) \cap I^{\prime}$ and $\operatorname{dist}\left(f_{y}(t), F(t, y(t))\right) \leq 2 N$ on $\left[t_{0}, \bar{t}+\theta\right) \cap\left(I \backslash I^{\prime}\right)$, which contradicts the maximality of $\left[t_{0}, \bar{t}\right)$. Hence $\bar{t}=T$.

It is clear that the pseudoderivative $f_{y}(\cdot)$ satisfies $\operatorname{dist}\left(f_{y}(t), F(t, y(t))\right)=k_{y}(t)$ with $k_{y}(t) \leq \varepsilon^{\prime}$ for every $t \in I^{\prime}$ and $k_{y}(t) \leq 2 N$ for every $t \in I \backslash I^{\prime}$. One checks easily that $\int_{I} k_{y}(t) d t \leq \varepsilon$. Hence, $y(\cdot)$ is an $\varepsilon$-solution of (1) on $I$.

The next lemma will play a crucial role in the sequel.

Lemma 2. Assume (H1)-(H3). Let $\varepsilon>0$ and let $x(\cdot)$ be an $\varepsilon$-solution of (1) on I. Then, there exist $l(\cdot)$ positive and bounded on I with $\int_{I} l(t) d t \leq 2 \varepsilon$ and $\eta>0$ such that for every $y_{0} \in \overline{D(A)}$ with $\left|x_{0}-y_{0}\right|<\eta$ we have that:

(i) the maximal solution $\tilde{v}(\cdot)$ of the scalar differential equation

$$
\dot{v}(t)=w(t, v(t))+l(t), v\left(t_{0}\right)=\left|x_{0}-y_{0}\right|,
$$

exists on I and 
(ii) for every $0<\delta<\varepsilon$ there exists a $\delta$-solution $y(\cdot)$ of (1) on I with $x_{0}$ replaced by $y_{0}$, satisfying

$$
|x(t)-y(t)| \leq \tilde{v}(t),
$$

for all $t \in I$.

Proof. The assertion (i) follows from ([13], Lemma 2.4) (see also Lemma 3 below).

Let $\varepsilon>0$ be fixed and let $f_{x}(\cdot)$ be the pseudoderivative of $x(\cdot)$. Then, due to Definition 3, $f_{x}(t) \in F(t, x(t)+\mathbb{B})+\mathbb{B}$ a.e. on $I$ and $k_{x}(t)=\operatorname{dist}\left(f_{x}(t), F(t, x(t))\right)$ satisfies $\int_{I} k_{x}(t) d t \leq \varepsilon$. Moreover, due to Remark $1, k_{x}(t) \leq 2 N$ for any $t \in I$.

We take $\varepsilon^{\prime} \leq \frac{\varepsilon}{5\left(T-t_{0}+N\right)}$. We can assume without loss of generality that there exists a compact set $I_{\varepsilon} \subset I$, with meas $\left(I \backslash I_{\varepsilon}\right)<\varepsilon^{\prime}$, such that the functions $\left.f_{x}\right|_{I_{\varepsilon}},\left.k_{x}\right|_{I_{\varepsilon}}$ and $\left.w\right|_{I_{\varepsilon} \times \mathbb{R}}$ are continuous.

Let $\delta<\varepsilon$. We can assume that there exists a compact set $I_{\delta} \subset I$ such that $I_{\varepsilon} \subset I_{\delta}$, meas $\left(I \backslash I_{\delta}\right)<\delta^{\prime}$, where $\delta^{\prime} \leq \min \left\{\frac{\delta}{5\left(T-t_{0}+N\right)}, \varepsilon^{\prime}\right\}$, and $\left.F\right|_{I_{\delta} \times X}$ is continuous.

We take $f_{x} \in F\left(t_{0}, x_{0}\right)$ such that $\left|f_{x}-f_{x}\left(t_{0}\right)\right| \leq k_{x}\left(t_{0}\right)+\varepsilon^{\prime}$. Let $\eta \in(0,1)$ and $y_{0} \in \overline{D(A)}$ with $\left|x_{0}-y_{0}\right|<\eta$. By (H3), there exists $f_{1} \in F\left(t_{0}, y_{0}\right)$ such that

$$
\left[x_{0}-y_{0}, f_{x}-f_{1}\right]_{+} \leq w\left(t_{0},\left|x_{0}-y_{0}\right|\right)+\varepsilon^{\prime} .
$$

Hence,

$$
\left[x_{0}-y_{0}, f_{x}\left(t_{0}\right)-f_{1}\right]_{+} \leq\left[x_{0}-y_{0}, f_{x}-f_{1}\right]_{+}+\left|f_{x}\left(t_{0}\right)-f_{x}\right| \leq w\left(t_{0},\left|x_{0}-y_{0}\right|\right)+2 \varepsilon^{\prime}+k_{x}\left(t_{0}\right) .
$$

Let $f(\cdot)$ be a Bochner integrable function such that $f(t) \in F\left(t, y_{0}\right)$ for every $t \in I$.

We consider the following cases.

Case 1. $t_{0}$ is a right dense point of $I_{\mathcal{E}}$ (hence it is a right dense point also for $I_{\delta}$ ).

We pick

$$
f_{y}(t)=\left\{\begin{array}{l}
f_{1}, \text { if } t \in I_{\delta} \\
f(t), \text { if } t \in I \backslash I_{\delta} .
\end{array}\right.
$$

Let $y^{1}(\cdot)$ be the integral solution of

$$
\dot{y}(t) \in A y(t)+f_{y}(t), y\left(t_{0}\right)=y_{0} .
$$

Then, by the continuity of $\left.F\right|_{I_{\delta} \times X}$ and $y^{1}(\cdot)$, there exists $\tau>t_{0}$ such that $f_{1} \in F\left(t, y^{1}(t)\right)+\delta^{\prime} \mathbb{B}$ for every $t \in\left[t_{0}, \tau\right) \cap I_{\delta}$.

Due to the continuity of $y^{1}(\cdot)$, the upper semicontinuity of $[\cdot, \cdot]_{+}$and the continuity of $w(\cdot, \cdot)$ at $\left(t_{0},\left|x_{0}-y_{0}\right|\right)$ and of $k_{x}(\cdot)$ at $t_{0}$, the number $\tau>t_{0}$ can be chosen such that $\left|y^{1}(t)-y_{0}\right| \leq \frac{1}{2}$ for every $t \in\left[t_{0}, \tau\right)$, and moreover,

$$
\begin{aligned}
& {\left[x(t)-y^{1}(t), f_{x}(t)-f_{1}\right]_{+} \leq\left[x_{0}-y_{0}, f_{x}\left(t_{0}\right)-f_{1}\right]_{+}+\varepsilon^{\prime}} \\
& \leq w\left(t_{0},\left|x_{0}-y_{0}\right|\right)+3 \varepsilon^{\prime}+k_{x}\left(t_{0}\right) \\
& \leq\left|w\left(t_{0},\left|x_{0}-y_{0}\right|\right)-w\left(t,\left|x(t)-y^{1}(t)\right|\right)\right|+w\left(t,\left|x(t)-y^{1}(t)\right|\right)+4 \varepsilon^{\prime}+k_{x}(t) \\
& \leq w\left(t,\left|x(t)-y^{1}(t)\right|\right)+5 \varepsilon^{\prime}+k_{x}(t)
\end{aligned}
$$

for every $t \in\left[t_{0}, \tau\right) \cap I_{\varepsilon}$.

Clearly, due to our choice of $\tau$, we have that $f_{y}(t) \in F\left(t, y^{1}(t)+\mathbb{B}\right)+\mathbb{B}$ for any $t \in\left[t_{0}, \tau\right)$, hence $y^{1}(\cdot)$ is a solution of $(5)$ on $\left[t_{0}, \tau\right)$. 
We set $y(t)=y^{1}(t)$ for any $t \in\left[t_{0}, \tau\right)$ and let $k_{y}(t)=\operatorname{dist}\left(f_{y}(t), F(t, y(t))\right)$. Then $k_{y}(t) \leq \delta^{\prime}$ for $t \in\left[t_{0}, \tau\right) \cap I_{\delta}$ and $k_{y}(t) \leq 2 N$ for $t \in\left[t_{0}, \tau\right) \cap\left(I \backslash I_{\delta}\right)$.

Hence, for any $t \in\left[t_{0}, \tau\right) \cap I_{\varepsilon}$,

$$
\left[x(t)-y(t), f_{x}(t)-f_{y}(t)\right]_{+} \leq w(t,|x(t)-y(t)|)+5 \varepsilon^{\prime}+k_{x}(t) .
$$

On the other hand, for any $t \in\left[t_{0}, \tau\right) \cap\left(I \backslash I_{\varepsilon}\right)$ we have that

$$
\left[x(t)-y(t), f_{x}(t)-f_{y}(t)\right]_{+} \leq\left|f_{x}(t)-f_{y}(t)\right| \leq 2 N \leq 2 N+w(t,|x(t)-y(t)|) .
$$

Case 2. $t_{0}$ is not a right dense point of $I_{\varepsilon}$ but it is a right dense point of $I_{\delta}$.

Let $y^{1}(\cdot)$ be the integral solution of $(9)$, where $f_{y}(\cdot)$ is chosen as in Case 1 . Then there exists $\tau>t_{0}$ such that $\left|y^{1}(t)-y_{0}\right| \leq \frac{1}{2}$ for every $t \in\left[t_{0}, \tau\right)$, and moreover, $\left[t_{0}, \tau\right) \subset I \backslash I_{\mathcal{E}}$. Moreover, we can choose $\tau$ such that $f_{1} \in F\left(t, y^{1}(t)\right)+\delta^{\prime} \mathbb{B}$ for every $t \in\left[t_{0}, \tau\right) \cap I_{\delta}$.

We set, as in the previous case, $y(t)=y^{1}(t)$ for any $t \in\left[t_{0}, \tau\right)$. Hence $k_{y}(t) \leq \delta^{\prime}$ for $t \in\left[t_{0}, \tau\right) \cap I_{\delta}$ and $k_{y}(t) \leq 2 N$ for $t \in\left[t_{0}, \tau\right) \cap\left(I \backslash I_{\delta}\right)$.

Case 3. $t_{0}$ is not a right dense point of $I_{\delta}$.

In this case, we let $y^{1}(\cdot)$ to be the integral solution of

$$
\dot{y}(t) \in A y(t)+f(t), y\left(t_{0}\right)=y_{0} .
$$

Then there exists $\tau>t_{0}$ such that $\left|y^{1}(t)-y_{0}\right| \leq \frac{1}{2}$ for every $t \in\left[t_{0}, \tau\right)$, and moreover, $\left[t_{0}, \tau\right) \subset I \backslash I_{\delta} \subset$ $I \backslash I_{\varepsilon}$. We have that $y^{1}(\cdot)$ is a solution of (5) on $\left[t_{0}, \tau\right)$.

We let $y(t)=y^{1}(t)$ and $f_{y}(t)=f(t)$ for every $t \in\left[t_{0}, \tau\right)$ and hence $k_{y}(t) \leq 2 N$ on $\left[t_{0}, \tau\right)$.

Moreover, in both cases 2 and 3, for any $t \in\left[t_{0}, \tau\right)$ we have that

$$
\left[x(t)-y(t), f_{x}(t)-f_{y}(t)\right]_{+} \leq 2 N+w(t,|x(t)-y(t)|) .
$$

We continue the above construction in a similar way by replacing $t_{0}$ by $\tau$ and $y_{0}$ by $y_{\tau}=\lim _{t \uparrow \tau} y(t)$.

Finally, reasoning as in the proof of Lemma 1, we define $y(\cdot)$ on $I$, solution of (5). Its pseudoderivative $f_{y}(\cdot)$ satisfies $\operatorname{dist}\left(f_{y}(t), F(t, y(t))\right)=k_{y}(t)$ with $k_{y}(t) \leq \delta^{\prime}$ for every $t \in I_{\delta}$ and $k_{y}(t) \leq 2 N$ for every $t \in I \backslash I_{\delta}$. One checks easily that $\int_{I} k_{y}(t) d t \leq \delta$. Hence, $y(\cdot)$ is a $\delta$-solution of (1) on $I$.

Moreover, for any $t \in I_{\mathcal{\varepsilon}}$, we have that

$$
\left[x(t)-y(t), f_{x}(t)-f_{y}(t)\right]_{+} \leq w(t,|x(t)-y(t)|)+5 \varepsilon^{\prime}+k_{x}(t)
$$

and, for any $t \in I \backslash I_{\varepsilon}$,

$$
\left[x(t)-y(t), f_{x}(t)-f_{y}(t)\right]_{+} \leq 2 N+w(t,|x(t)-y(t)|) .
$$

Furthermore, using (3), we have that

$$
\begin{gathered}
|x(t)-y(t)| \leq\left|x_{0}-y_{0}\right|+\int_{t_{0}}^{t}\left[x(s)-y(s), f_{x}(s)-f_{y}(s)\right]_{+} d s \\
\leq\left|x_{0}-y_{0}\right|+\int_{\left[t_{0}, t\right] \cap I_{\varepsilon}}\left(w(s,|x(s)-y(s)|)+5 \varepsilon^{\prime}+k_{x}(s)\right) d s \\
\quad+\int_{\left[t_{0}, t\right] \cap\left(I \backslash I_{\varepsilon}\right)}(w(s,|x(s)-y(s)|)+2 N) d s
\end{gathered}
$$




$$
\leq\left|x_{0}-y_{0}\right|+\int_{t_{0}}^{t} w(s,|x(s)-y(s)|) d s+\int_{\left[t_{0}, t\right] \cap I_{\varepsilon}}\left(5 \varepsilon^{\prime}+k_{x}(s)\right) d s+\int_{\left[t_{0}, t\right] \cap\left(I \backslash I_{\varepsilon}\right)} 2 N d s
$$

for any $t \in I$. Let $l(t)=5 \varepsilon^{\prime}+k_{x}(t)$ for $t \in I_{\varepsilon}$ and $l(t)=2 N$ for $t \in I \backslash I_{\varepsilon}$. Then, for any $t \in I$,

$$
|x(t)-y(t)| \leq\left|x_{0}-y_{0}\right|+\int_{t_{0}}^{t} w(s,|x(s)-y(s)|) d s+\int_{t_{0}}^{t} l(s) d s .
$$

Hence, $|x(t)-y(t)| \leq \tilde{v}(t)$ for every $t \in I$, where $\tilde{v}(\cdot)$ is the maximal solution of the scalar differential equation

$$
\dot{v}(t)=w(t, v(t))+l(t), v\left(t_{0}\right)=\left|x_{0}-y_{0}\right|
$$

on $I$. Clearly, $l(\cdot)$ is bounded on $I$ and

$$
\int_{I} l(s) d s=\int_{I_{\varepsilon}}\left(5 \varepsilon^{\prime}+k_{x}(s)\right) d s+\int_{I \backslash I_{\varepsilon}} 2 N d s \leq 5 \varepsilon^{\prime}\left(T-t_{0}\right)+\varepsilon+2 N \varepsilon^{\prime} \leq 2 \varepsilon .
$$

The proof is completed.

The proof of the following result follows the same steps as the proof of ([13], Lemma 2.4) and it is omitted.

Lemma 3. Let $\lambda \in L^{1}\left(I ; \mathbb{R}_{+}\right)$and let $v: I \times \mathbb{R}_{+} \rightarrow \mathbb{R}_{+}$be a Carathéodory function, integrally bounded on the bounded sets, with $v(t, \cdot)$ nondecreasing for every $t \in I$. If the maximal solution $h(\cdot)$ of $(7)$ exists on I, then for every $\varepsilon>0$ there exists $\delta>0$ such that the maximal solution $\bar{r}(\cdot)$ of

$$
\dot{r}(t)=v(t, r(t))+\mu(t), r\left(t_{0}\right)=\bar{r}_{0} \in\left[r_{0}, r_{0}+\delta\right],
$$

exists on $I$ and $\bar{r}(t) \leq h(t)+\varepsilon$ on $I$, for every function $\mu(\cdot)$ such that $0 \leq \mu(t) \leq \lambda(t)$ for $t \in I$ and $\int_{I} \mu(t) d t \leq \delta$

Now, by using the previous lemmas, we will prove the following existence result of a limit solution for the Cauchy problem (1).

Theorem 1. Assume (H1)-(H3). Let $\varepsilon>0$ and let $x(\cdot)$ be an $\varepsilon$-solution of (1). Then, there exist a positive and bounded function $l(\cdot)$ with $\int_{I} l(t) d t \leq 2 \varepsilon$ and $\eta>0$ such that for every $y_{0} \in \overline{D(A)}$ with $\left|x_{0}-y_{0}\right|<\eta$ we have that:

(i) the maximal solution $\tilde{v}(\cdot)$ of the scalar differential equation

$$
\dot{v}(t)=w(t, v(t))+l(t), v\left(t_{0}\right)=\left|x_{0}-y_{0}\right|,
$$

exists on I and

(ii) there exists a limit solution $y(\cdot)$ of (1) on I with $y\left(t_{0}\right)=y_{0}$ such that

$$
|x(t)-y(t)| \leq \tilde{v}(t)+\varepsilon,
$$

for every $t \in I$.

Proof. Let $\delta>0$ be given by Lemma 3, corresponding to $\varepsilon / 2$. Take $\varepsilon_{1} \leq \min \{\varepsilon / 2, \delta / 2\}$. By Lemma 2 there exist $l_{1}(\cdot)$ a positive and bounded function with $\int_{I} l_{1}(t) d t \leq 2 \varepsilon$ and $\eta>0$ such that for any $y_{0} \in \overline{D(A)}$ with $\left|x_{0}-y_{0}\right|<\eta$ there exists $y_{1}(\cdot)$ an $\varepsilon_{1}$-solution of (1) with $y_{1}\left(t_{0}\right)=y_{0}$ satisfying

$$
\left|x(t)-y_{1}(t)\right| \leq v_{1}(t)
$$


where $v_{1}(\cdot)$ is the maximal solution of

$$
\dot{v}(t)=w(t, v(t))+l_{1}(t), v\left(t_{0}\right)=\left|x_{0}-y_{0}\right|
$$

on $I$.

Let $\delta_{1}>0$ be given by Lemma 3 corresponding to $\varepsilon_{1} / 2$. Take $\varepsilon_{2} \leq \min \left\{\varepsilon_{1} / 2, \delta_{1} / 2\right\}$. By Lemma 2 there exists an $\varepsilon_{2}$-solution $y_{2}(\cdot)$ of (1) on $I$ with $y_{2}\left(t_{0}\right)=y_{0}$ such that

$$
\left|y_{2}(t)-y_{1}(t)\right| \leq v_{2}(t),
$$

for every $t \in I$. Here $v_{2}(\cdot)$ is the maximal solution of

$$
\dot{v}(t)=w(t, v(t))+l_{2}(t), v\left(t_{0}\right)=0,
$$

where $l_{2}(\cdot)$ is positive and bounded on $I$ and $\int_{I} l_{2}(t) d t \leq 2 \varepsilon_{1} \leq \delta$. Then, by Lemma $3, v_{2}(t) \leq \varepsilon / 2$ for any $t \in I$.

We construct by induction a sequence of $\varepsilon_{n}$-solutions $\left(y_{n}(\cdot)\right)$ of (1) on $I$, where $\varepsilon_{n} \leq$ $\min \left\{\varepsilon_{n-1} / 2, \delta_{n-1} / 2\right\}$, for any $n=2,3, \ldots$, such that

$$
\left|y_{n+1}(t)-y_{n}(t)\right| \leq v_{n+1}(t),
$$

for every $t \in I$. Here $v_{n+1}(\cdot)$ is the maximal solution of

$$
\dot{v}(t)=w(t, v(t))+l_{n+1}(t), v\left(t_{0}\right)=0,
$$

where $l_{n+1}(\cdot)$ is positive and bounded on $I$ and satisfies $\int_{I} l_{n+1}(t) d t \leq 2 \varepsilon_{n} \leq \delta_{n-1}$. Moreover, $v_{n+1}(t) \leq \varepsilon_{n-1} / 2$ for every $t \in I$ and every $n=2,3, \ldots$ Therefore,

$$
\left|y_{n+1}(t)-y_{n}(t)\right| \leq \varepsilon_{n-1}
$$

for every $t \in I$ and every $n=2,3, \ldots$. Taking into account that $\sum_{n=1}^{\infty} \varepsilon_{n} \leq \varepsilon$, we conclude that $\left(y_{n}(\cdot)\right)$ is a Cauchy sequence in $C(I ; X)$. Thus, there exists a continuous function $y: I \rightarrow X$ such that $\lim _{n \rightarrow \infty} y_{n}(t)=y(t)$ uniformly on $I$. Furthermore, $|x(t)-y(t)| \leq v_{1}(t)+\varepsilon$, where $v_{1}(\cdot)$ is the maximal solution of (11).

The next theorem is a variant of the well known lemma of Filippov-Pliś. This lemma has numerous applications in optimal control theory and had been proved on different variants by different authors. In the next theorem, we extend this result to the case when the integral solutions do not necessarily exist. Variants of this lemma have been proved in $[14,15]$ for the case of uniformly convex dual space and in [16] for the case when $A$ generates a compact semigroup.

Theorem 2. Assume (H1)-(H3). Let $x(\cdot)$ be an integral solution of the differential inclusion

$$
\left\{\begin{array}{l}
\dot{x}(t) \in A x(t)+F(t, x(t))+g(t) \mathbb{B}, \\
x\left(t_{0}\right)=x_{0} \in \overline{D(A)}
\end{array}\right.
$$

on $I$, where $g \in L^{1}\left(I ; \mathbb{R}_{+}\right)$. Then for every $\varepsilon>0$ and every $y_{0} \in \overline{D(A)}$ for which the maximal solution $v(\cdot)$ of the scalar differential equation

$$
\dot{v}(t)=w(t, v(t))+g(t), v\left(t_{0}\right)=\left|x_{0}-y_{0}\right|,
$$


exists on $I$, there exists a limit solution $z(\cdot)$ of $(1)$ on I with $z\left(t_{0}\right)=y_{0}$, satisfying

$$
|x(t)-z(t)| \leq v(t)+\varepsilon,
$$

for all $t \in I$.

Proof. Let $f_{x}(\cdot)$ be the pseudoderivative of $x(\cdot)$. Then $f_{x}(t) \in F(t, x(t))+g(t) \mathbb{B}$ for every $t \in I$. Furthermore, for every $\varepsilon>0$ there exists a compact $I_{\mathcal{E}} \subset I$ with Lebesgue measure meas $\left(I \backslash I_{\mathcal{E}}\right)<\varepsilon$ such that $\left.f_{x}\right|_{I_{\varepsilon}},\left.g\right|_{I_{\varepsilon}},\left.F\right|_{I_{\varepsilon} \times X}$ and $\left.w\right|_{I_{\varepsilon} \times \mathbb{R}_{+}}$are continuous. We fix $v>0$ and define the multifunction

$$
G(t, u)=\overline{\left\{v \in F(t, u) ;\left[x(t)-u, f_{x}(t)-v\right]_{+}<w(t,|x(t)-u|)+g(t)+v\right\}} .
$$

It follows from $(\mathrm{H} 3)$ that $G(\cdot, \cdot)$ has nonempty closed values. Moreover, $G(\cdot, \cdot)$ is almost LSC (the proof follows, with obvious modifications, the same lines as the proof of ([16], Theorem 2). Due to Lemma 1, for every $\mu>0$ the evolution inclusion

$$
\left\{\begin{array}{l}
\dot{x}(t) \in A x(t)+G(t, x(t)) \\
x\left(t_{0}\right)=y_{0}
\end{array}\right.
$$

has a $\mu$-solution $y(\cdot)$ defined on the whole $I$. Then, its pseudoderivative $f_{y}(\cdot)$ satisfies $f_{y}(t) \in$ $G(t, y(t))+h_{y}(t) \mathbb{B}$ for any $t \in I$, where $h_{y}(t) \leq 2 N$ on $I$ and $\int_{I} h_{y}(s) d s \leq \mu$. It follows from the properties of $[\cdot, \cdot]+$ that

$$
\left[x(t)-y(t), f_{x}(t)-f_{y}(t)\right]_{+} \leq w(t,|x(t)-y(t)|)+g(t)+v+h_{y}(t) .
$$

Thus, $|x(t)-y(t)| \leq r(t)$, where $r(\cdot)$ is the maximal solution of the inequality $\dot{r}(t) \leq w(t, r(t))+g(t)+$ $v+h_{y}(t)$ with $r\left(t_{0}\right)=\left|x_{0}-y_{0}\right|$.

Due to Lemma $3, r(\cdot)$ exists on the whole $I$ for sufficiently small $\nu$ and $\mu$ and moreover, for every $\varepsilon>0$ there exists $\kappa>0$ such that $r(t) \leq v(t)+\varepsilon$ for $\mu, v<\kappa$.

Clearly, $y(\cdot)$ is a $\mu$-solution also of (1). It follows from Theorem 1 that there exists a limit solution $z(\cdot)$ of (1) such that $|z(t)-y(t)| \leq \varepsilon$. The proof is therefore complete thanks to the triangle inequality.

Remark 2. In fact, Theorem 2 says that the solution set of (1) depends continuously on small perturbations of the initial condition and the right-hand side.

\subsection{Limit and Integral Solutions}

We start this subsection by giving a simple example to illustrate the notion of limit solutions.

Example 1. Let $A \equiv 0$. We consider the ordinary differential inclusion:

$$
\dot{x}(t) \in \mathbb{B}, t \in(0,1), x(0)=0 .
$$

Here $\mathbb{B}$ denotes the unit ball in $L^{1}\left(0,1 ; \mathbb{R}^{n}\right)$. Clearly, the limit solutions of $(14)$ are all Lipschitz functions (of Lipschitz constant 1). However, there exists such kind of functions nowhere differentiable, i.e., which are not integral solutions.

First, we will prove that the set of limit solutions is the closure of the set of integral solutions of (1) when $F(\cdot, \cdot)$ satisfies the following stronger assumption than $(\mathrm{H} 3)$. 
Hypothesis $\mathbf{3}^{\prime}\left(\mathbf{H} \mathbf{3}^{\prime}\right)$. (Full Perron condition) There exists a Perron function $w(\cdot, \cdot)$ such that

$$
D_{H}(F(t, x), F(t, y)) \leq w(t,|x-y|)
$$

for every $x, y \in X$ and every $t \in I$.

Theorem 3. Assume (H1), (H2) and (H3'). Then (1) has integral solutions. Furthermore, the set of integral solutions of (1) is dense in the set of limit solutions of (1).

Proof. Let $\varepsilon>0$ and let $y(\cdot)$ be an $\varepsilon$-solution (1) with the pseudoderivative $f_{y}(\cdot)$. Then $f_{y}(t) \in$ $F(t, y(t))+h_{y}(t) \mathbb{B}$ for any $t \in I$, where $h_{y}(t) \leq 2 N$ on $I$ and $\int_{I} h_{y}(t) d t \leq \varepsilon$.

Let $0<\delta<\varepsilon$. Since the function $w(\cdot, \cdot)$ is Perron, there exists $0<\mu<\varepsilon$ such that $\int_{I} w(t, \mu) d t<\delta$. Furthermore, there exists $t_{1}>t_{0}$ such that $\left|y(t)-x_{0}\right|<\mu$ for $t \in\left[t_{0}, t_{1}\right]$. Let $z(t):=y(t)$ on $\left[t_{0}, t_{1}\right)$ and denote $z^{1}=z\left(t_{1}\right)$. By $(\mathrm{H} 1)$ and $\left(\mathrm{H}^{\prime}\right)$, there exists a strongly measurable function $f_{1}(\cdot)$ such that $f_{1}(t) \in F\left(t, z^{1}\right)$ and

$$
\left|f_{y}(t)-f_{1}(t)\right| \leq w\left(t,\left|y(t)-z^{1}\right|\right)+h_{y}(t)+\mu
$$

a.e. on $\left[t_{1}, T\right]$. Consider the problem

$$
\left\{\begin{array}{l}
\dot{z}(t) \in A z(t)+f_{1}(t) \\
z\left(t_{1}\right)=z^{1}
\end{array}\right.
$$

and let $z_{1}(\cdot)$ be a solution of (15) on $\left[t_{1}, T\right]$. There exists $t_{2}>t_{1}$ such that $\left|z_{1}(t)-z^{1}\right|<\mu$ for any $t \in\left[t_{1}, t_{2}\right]$. Then, on $\left[t_{1}, t_{2}\right]$,

$$
\left|f_{y}(t)-f_{1}(t)\right| \leq w\left(t,\left|y(t)-z_{1}(t)\right|\right)+\mid w\left(t,\left|y(t)-z_{1}(t)\right|+\mu\right)-w\left(t,\left|y(t)-z_{1}(t)\right|\right)+h_{y}(t)+\mu .
$$

Denote $M_{w}(\mu):=\sup _{|x| \leq 2 N}|w(t,|x|+\mu)-w(t,|x|)|$ and let $z(t):=z_{1}(t)$ on $\left[t_{1}, t_{2}\right]$. Then, $z(\cdot)$ is a solution of $\dot{z}(t) \in A z(t)+F(t, z(t))+w(t, \mu) \mathbb{B}$ and

$$
\left|f_{y}(t)-f_{z}(t)\right| \leq w(t,|y(t)-z(t)|)+M_{w}(\mu)+h_{y}(t)+\mu
$$

on $\left[t_{1}, t_{2}\right]$.

Using the same method as above, as in the proof of Lemma 1, we can extend $z(\cdot)$ on the whole interval $I$, such that $\dot{z}(t) \in A z(t)+F(t, z(t))+w(t, \mu) \mathbb{B}$ and

$$
\left|f_{y}(t)-f_{z}(t)\right| \leq w(t,|y(t)-z(t)|)+M_{w}(\mu)+h_{y}(t)+\mu
$$

for any $t \in I$. Moreover, $\int_{I} \operatorname{dist}\left(f_{z}(t), F(t, z(t))\right) d t \leq \int_{I} w(t, \mu)<\delta$ on $I$. Hence, $z(\cdot)$ is a $\delta$-solution of (1). Using (4), we get that $|y(t)-z(t)| \leq r(t)$, where $r(\cdot)$ is the maximal solution of

$$
\dot{r}(t) \leq w(t, r(t))+M_{w}(\varepsilon)+h_{y}(t)+\varepsilon, r\left(t_{0}\right)=0 .
$$

Now, let $\varepsilon_{n} \downarrow 0$ and let $\left(x_{n}(\cdot)\right)$ be a sequence of $\varepsilon_{n}$-solutions of (1), constructed as above, with $\left(f_{n}(\cdot)\right)$ the corresponding sequence of pseudoderivatives. Then

$$
\left|x_{n}(t)-x_{n+1}(t)\right| \leq r_{n}(t)
$$

and

$$
\left|f_{n}(t)-f_{n+1}(t)\right| \leq w\left(t, r_{n}(t)\right)+M_{w}\left(\varepsilon_{n}\right)+h_{n+1}(t)+\varepsilon_{n}
$$


where $r_{n}(\cdot)$ is the maximal solution of

$$
\dot{r}_{n}(t) \leq w\left(t, r_{n}(t)\right)+M_{w}\left(\varepsilon_{n}\right)+h_{n+1}(t), r_{n}\left(t_{0}\right)=0 .
$$

Due to the definitions of $M_{w}\left(\varepsilon_{n}\right)$ and since $w(\cdot, \cdot)$ is Perron, one can choose $\left(\varepsilon_{n}\right)$ such that $\sum_{n=1}^{\infty}\left|x_{n}(t)-x_{n+1}(t)\right|$ converges uniformly to 0 and $\left(f_{n}(\cdot)\right)$ converges $L^{1}$-strongly. Therefore, $\lim _{n \rightarrow \infty} x_{n}(t)=x(t)$ and $\lim _{n \rightarrow \infty} f_{n}(t)=f(t)$. Then $f(t) \in F(t, x(t))$ since $F(\cdot, \cdot)$ is almost continuous and $\dot{x}(t) \in A x(t)+f(t)$ with $x\left(t_{0}\right)=x_{0}$. Therefore, $x(\cdot)$ is an integral solution of (1).

To prove the second part of the theorem, let $\delta>0$. Let $z(\cdot)$ be a limit solution of (1). Therefore, for any $\varepsilon>0$ there exists an $\varepsilon$-solution $z_{\varepsilon}(\cdot)$ such that $\left|z(t)-z_{\varepsilon}(t)\right|<\varepsilon$ for $t \in I$. As in the first part of the proof starting from $z_{\varepsilon}(\cdot)$, we can choose $\varepsilon_{n} \downarrow 0$ with $\varepsilon_{1}=\varepsilon$ such that there exists an integral solution $x(\cdot)$ of (1) with $\left|x(t)-z_{\varepsilon}(t)\right|<\delta$ on $I$. Hence, $|z(t)-x(t)|<\varepsilon+\delta$ for any $t \in I$. The proof is completed.

We refer the reader to ([4], pp. 25-27), where the author gives one example of nonexistence of solutions even when $X=\mathbb{R}^{n}$. In this case, the set of limit solutions is nonempty and closed.

In [4] it is also studied another example where the solution set of

$$
\dot{x}(t) \in A x(t)+K, x\left(t_{0}\right)=x_{0} \in \overline{D(A)},
$$

with $K$ convex compact, is not closed. In this case, since the multivalued term is constant, due to Theorem 3, the set of integral solutions is nonempty and dense in the set of limit solutions.

Remark 3. Consider the relaxed problem (6). The solution set of this problem is not closed, in general. We are not able to prove that it is contained in the set of limit solutions of $(1)$, even if $F(t, \cdot)$ is Lipshitz continuous. Nevertheless, if the solution set of (1) is dense in the solution set of (6), then every relaxed solution is also a limit solution. We refer the reader to [16,17], where this type of relaxation theorems are proved in Banach spaces with some additional properties. In our opinion, the limit solution set is more adequate, because it is compact and, under mild assumptions, it is the closure of the solution set of (1).

Definition 4. (see, e.g., [18]) The m-dissipative operator $A$ is said to be of complete continuous type if for every $a<b$ and every $\left(f_{n}(\cdot)\right)$ in $L^{1}(a, b ; X)$ and $\left(x_{n}(\cdot)\right)$ in $C([a, b], X)$, with $x_{n}(\cdot)$ a solution on $[a, b]$ of $\dot{x}_{n}(t) \in A x_{n}(t)+f_{n}(t), n=1,2, \ldots, \lim _{n \rightarrow \infty} f_{n}=f$ weakly in $L^{1}(a, b ; X)$ and $\lim _{n \rightarrow \infty} x_{n}=x$ uniformly in $C([a, b], X)$, it follows that $x$ is a solution on $[a, b]$ of

$$
\dot{x}(t) \in A x(t)+f(t) .
$$

We need the following assumption:

Hypothesis $4 \mathbf{( H 4 )} . F(\cdot, \cdot)$ has nonempty convex weakly compact values.

We give now sufficient conditions that the limit solutions to be integral ones.

Theorem 4. Let A be of complete continuous type. If (H1)-(H4) hold, then every limit solution of (1) is also an integral solution of (1).

Proof. Let $\left(x_{n}(\cdot)\right)$ be a sequence of $\varepsilon_{n}$-solutions of (1) with $\varepsilon_{n} \downarrow 0$ such that $\lim _{n \rightarrow \infty} x_{n}(t)=x(t)$ uniformly on I. Consequently, the set $\mathcal{M}=\bigcup_{t \in I} \bigcup_{n=1}^{\infty}\left\{x_{n}(t)\right\}$ is compact. Denote by $\left(f_{n}(\cdot)\right)$ the corresponding sequence of pseudoderivatives, hence $\int_{I} \operatorname{dist}\left(f_{n}(t), F\left(t, x_{n}(t)\right)\right) d t \leq \varepsilon_{n}$ for any natural $n$. Let $\bar{f}_{n}(\cdot) \in$ 
$L^{1}(I ; X)$ be such that $\bar{f}_{n}(t) \in F\left(t, x_{n}(t)\right)$ and $\left|f_{n}(t)-\bar{f}_{n}(t)\right| \leq \frac{3}{2} \operatorname{dist}\left(f_{n}(t), F\left(t, x_{n}(t)\right)\right)$ for a.a. $t \in I$. Take $y_{n}(\cdot)$ the solutions of

$$
\dot{y}_{n}(t) \in A y_{n}(t)+\bar{f}_{n}(t), y_{n}\left(t_{0}\right)=x_{0} .
$$

Due to (4), $\left|x_{n}(t)-y_{n}(t)\right| \leq \int_{t_{0}}^{t}\left|f_{n}(t)-\bar{f}_{n}(t)\right| d t \leq \frac{3}{2} \varepsilon_{n}$. Consequently, $\left(y_{n}(\cdot)\right)$ converges uniformly to $x(\cdot)$.

On the other hand, since $F(\cdot, \cdot)$ is almost continuous, for any $\varepsilon>0$ there exists a compact set $I_{\varepsilon} \subset I$ with meas $\left(I \backslash I_{\mathcal{E}}\right) \leq \varepsilon$ such that $\left.F\right|_{I_{\varepsilon} \times X}$ is continuous. Therefore, $F: I_{\mathcal{E}} \times X \rightrightarrows X_{w}$ is also continuous. Here $X_{w}$ is $X$ endowed with the weak topology. Due to (H4), the set $K_{\varepsilon}:=\overline{c o}\left(\bigcup_{t \in I_{\varepsilon}} \bigcup_{n=1}^{\infty} F\left(t, x_{n}(t)\right)\right)$ is weakly compact. We have that $\bar{f}_{n}(t) \in K_{\varepsilon}$ on $I_{\varepsilon}$. Moreover, since $\left(\bar{f}_{n}(\cdot)\right)$ is uniformly integrable, it is relatively weakly compact. Then, passing to subsequences, $\bar{f}_{n}(\cdot) \rightarrow f(\cdot)$ weakly in $L^{1}(I ; X)$. Moreover, as $F(\cdot, \cdot)$ is almost continuous, $f(t) \in F(t, x(t))$ a.e. on $I$.

Finally, since $A$ is of complete continuous type, we get that $x(\cdot)$ is the solution of

$$
\dot{x}(t) \in A x(t)+f(t), x\left(t_{0}\right)=x_{0} .
$$

The proof is therefore complete.

\section{3. m-Dissipative Inclusions with Compact Semigroup}

In this section, we will study the differential inclusion (1) under the following additional assumption on $A$.

(A) The semigroup $\{S(\cdot) ; t \geq 0\}$ is compact, i.e., $S(t)$ is a compact operator for every $t>0$.

Since $\|F(t, x(T))\| \leq N$ for every solution $x(\cdot)$ of (5) the following result is a consequence of ([4], Lemma 3.1).

Lemma 4. Under hypotheses (H1)-(H3) and (A), the set of integral solutions of (1) is $C(I, X)$ precompact (if nonempty).

Notice also the following theorem which is proved in [19].

Theorem 5. Let $F(\cdot, \cdot)$ be almost LSC with closed bounded values and let X be a separable Banach space. Under hypotheses (H2) and (A), the set of integral solutions of (1) is nonempty.

As a corollary, one can prove the following variant of Filippov-Pliś Lemma (see ([16], Theorem 3) for the separable case).

Proposition 1. Assume (H1)-(H3) and (A). Let $x(\cdot)$ be an integral solution of the Cauchy problem

$$
\dot{x}(t) \in A x(t)+f_{x}(t), x\left(t_{0}\right)=x_{0} \in \overline{D(A)},
$$

on $I$, where $\operatorname{dist}\left(f_{x}(t) ; F(t, x(t))\right) \leq g(t)$ for all $t \in I$ and $g \in L^{1}\left(t_{0}, T ; \mathbb{R}^{+}\right)$. Then for any $\varepsilon>0$ and any $y_{0} \in \overline{D(A)}$, there exists a solution $y(\cdot)$ of the Cauchy problem (1) on I with $x_{0}$ replaced by $y_{0}$ such that

$$
|x(t)-y(t)| \leq v(t)+\varepsilon,
$$

for all $t \in I$, where $v(\cdot)$ is the maximal solution of the scalar differential equation $\dot{v}(t)=w(t, v(t))+$ $g(t), v\left(t_{0}\right)=\left|x_{0}-y_{0}\right|$, on $I$.

We are ready to prove the following interesting result. 
Theorem 6. Under hypotheses (H1)-(H3) and (A), the set of integral solutions of (1) is dense in the set of limit solutions of (1).

Proof. Let $x(\cdot)$ be a limit solution of (1) on $I$. Then there exists a sequence $\left(x_{n}(\cdot)\right)$ of $\varepsilon_{n}$-solutions of (1) with $\varepsilon_{n} \downarrow 0$ such that $\lim _{n \rightarrow \infty}\left|x_{n}(t)-x(t)\right|=0$ uniformly on $I$. Then, for any natural $n, x_{n}(\cdot)$ is a solution of $\dot{x}_{n}(t) \in A x_{n}(t)+f_{n}(t)$, where $\operatorname{dist}\left(f_{n}(t) ; F\left(t, x_{n}(t)\right)=g_{n}(t)\right.$ with $0<g_{n}(t) \leq 2 N$ on $I$ and $\int_{I} g_{n}(t) d t \leq \varepsilon_{n}$. Due to Proposition 1 , to every $n$ there exists a solution $y_{n}(\cdot)$ of (1) such that

$$
\left|x_{n}(t)-y_{n}(t)\right| \leq v_{n}(t)+\frac{\varepsilon}{2^{n}}
$$

where $v_{n}(\cdot)$ is the maximal solution of the scalar differential equation $\dot{v}(t)=w(t, v(t))+g_{n}(t), v\left(t_{0}\right)=0$, on I. From Lemma 1, we have that $\lim _{n \rightarrow \infty} v_{n}(t)=0$ uniformly on $I$. Consequently, $\lim _{n \rightarrow \infty}\left|x_{n}(t)-y_{n}(t)\right|=0$ uniformly on $I$, i.e., $x(t)=\lim _{n \rightarrow \infty} y_{n}(t)$ uniformly on $I$.

\subsection{Example}

The following example is a modification of ([20], Example) and ([16], Example 1).

Let $\Omega \subset \mathbb{R}^{n}$ with $n \geq 4$ be a domain with smooth boundary $\partial \Omega$. Define $\varphi(r)=|r|^{\gamma-1} r$ for $r \neq 0$ and $0<\gamma<\frac{n-2}{n}$. We consider the following system:

$$
\left\{\begin{array}{l}
u_{t} \in \Delta \varphi(u)+G(t, y, u) \\
-\frac{\partial \varphi(u)}{\partial v} \in \beta(u) \text { on }(0, T) \times \partial \Omega \\
u(0, y)=u_{0}(y) .
\end{array}\right.
$$

Here, $u \in \mathbb{R}, \frac{\partial \varphi(u)}{\partial v}$ is the outward normal derivative on $\partial \Omega$ and $\beta(\cdot)$ is a maximal monotone graph in $\mathbb{R}$ with $\beta(0) \ni 0$. The multifunction $G$ has nonempty compact values, is measurable on all variables and continuous on the third one.

Define the operator $B$ in $L^{1}(\Omega)$ by

$$
\begin{gathered}
B u=\Delta \varphi(u), \text { for } u \in D(B), \text { where } \\
D(B)=\left\{u \in L^{1}(\Omega) ; \varphi(u) \in W^{1,1}(\Omega), \Delta \varphi(u) \in L^{1}(\Omega),-\frac{\partial \varphi(u)}{\partial v} \in \beta(u) \text { on } \partial \Omega\right\} .
\end{gathered}
$$

The derivatives here are understood in the sense of distributions.

As it is shown in ([4], p. 97), the operator $B$ defined above is m-dissipative in $L^{1}(\Omega)$ and generates a noncompact semigroup. Notice that in [4] the author works with m-accretive operators $A$; however $A$ is $\mathrm{m}$-dissipative iff $-A$ is $\mathrm{m}$-accretive.

Let

$$
F(t, x)=\left\{f \in L^{1}(\Omega) ; f(y) \in G(t, y, x(t, y)) \text { a.e. in } \Omega\right\},
$$

which is jointly measurable and continuous on $x$. We assume also that there exists $h \in L^{1}([0, T])$ such that $\|F(t, x)\| \leq h(t)(1+|x|)$. Let $x_{0}=u(\cdot) \in D(B)$. Therefore (H1), (H2) hold true.

Suppose also that there exists a Perron function $w(\cdot, \cdot)$ such that for every $x, z \in \Omega$ and every $f \in F(t, x)$ there exists $g \in F(t, z)$ such that

$$
\begin{array}{r}
\int_{\Omega^{+}(x \rightarrow z)}(f(y)-g(y)) d y-\int_{\Omega^{-}(x \rightarrow z)}(f(y)-g(y)) d y \\
\pm \int_{\Omega^{0}(x \rightarrow z)}(f(y)-g(y)) d y \leq w\left(t, \int_{\Omega}|f(y)-g(y)|\right) d y .
\end{array}
$$


Here, $\Omega_{x \rightarrow y}^{+(-, 0)}=\{y \in \Omega ; f(y)>g(y)(<,=)\}$. It follows from the characterization of $[\cdot, \cdot]_{+}$(see, e.g., [21], Example 1.4.3) that (H3) also hold true.

In the case when $\gamma>\frac{n-2}{n}$ the operator $B$ generates a compact semigroup and it is of complete continuous type.

\subsection{Applications to Optimal Control}

Our results can be applied to the following optimal control problem:

$$
\min \left\{g(x(T))+\int_{t_{0}}^{T} f(t, x(t)) d t\right\},
$$

where $x(\cdot)$ is a solution of $(1)$. Here, $f(\cdot, \cdot)$ is Carathéodory and integrally bounded on the bounded sets and the function $g: X \rightarrow \mathbb{R}$ is assumed to be lower semicontinuous.

Assume (H1)-(H3) and (A). In this case, the limit solution set of (1) is compact and moreover, the set of integral solutions of (1) is dense in the set of limit solutions (see Theorem 6 and Lemma 4).

Clearly, in general, the problem (16) has no optimal solution.

Theorem 7. Under the above conditions, the problem (16) admits an optimal limit solution.

Proof. The functional $x(\cdot) \rightarrow \int_{t_{0}}^{T} f(t, x(t)) d t$ is continuous from $C(I, X)$ into $\mathbb{R}$. Furthermore, $x(\cdot) \rightarrow$ $g(x(T))$ is lower semicontinuous. Consequently, the functional $J(x(\cdot))=g(x(T))+\int_{t_{0}}^{T} f(t, x(t)) d t$ is lower semicontinuous from $C(I, X)$ into $\mathbb{R}$. The proof follows from the facts that the limit solution set is $C(I, X)$ compact and every lower semicontinuous real valued function attains its minimum on a compact set.

\section{Conclusions}

As we pointed out, the theory of parabolic differential equations and inclusions written in the abstract operator form is growing rapidly. We refer the reader to [1-3] for the theory of PDE and their investigations as abstract equations. Especially the multivalued evolution equations are comprehensively studied in $[4,5,18]$. In the book by [5], the authors study differential inclusions in evolution (Gelfand) triple. The authors provide many interesting results and examples. In that case, the compactness assumptions are crucially used. In [17], the author prove relaxation theorem in that case.

In [4], the author restricted the study to Banach spaces with uniformly convex duals and $A$ generating a compact semigroup, or he used compactness-type assumptions regarding the Kuratowski (or Hausdorff) measure of noncompactness. In that case, every limit solution is also an integral one. That implies that our existence results extend the existence result there. Notice also [19] where lower semicontinuous perturbations of $\mathrm{m}$-dissipative operators are considered. The existence theorem there is used in the proof of Theorem 6 in this paper. We recall also the book by [18], devoted to nonlocal problems of evolution inclusions with time lag. The main assumptions there are that $A$ is completely continuous and generates a compact semigroup. We mention also [22] where functional evolution inclusions are studied.

In [12], the author uses full Perron condition in the case of ordinary differential inclusions in Banach spaces. The author assumes that the multifunction $F$ has strongly compact values.

The one-sided Perron condition as used here was introduced in [23]. Using integral representation of the solutions the author defined the so-called weak solutions (which are developed in [8]). Here the integral representation of the solution does not hold when $A$ is nonlinear and we use limit solutions. The case of a Banach space with uniformly convex dual was studied in [13] where it was shown that if $F$ has compact values, then the solution set of (1) is compact $R_{\delta}$ and a relaxation theorem has 
been proved. No other compactness conditions were used. The paper [14] was devoted to Lemma of Filippov-Pliś. The papers $[15,16]$ study the problem (1) in the case when the Banach space has uniformly convex dual.

In the present paper we introduce the so-called limit solutions for the fully nonlinear evolution inclusion (1) and we study their properties. In general, the limit solutions of (1) are not solutions of the relaxed system (6).

(a) The set of limit solutions is nonempty and always $C(I, X)$ closed when the right hand side $F$ is almost continuous with closed bounded values and one-sided Perron in the state variable. Furthermore, every integral solution is also a limit solution.

(b) The set of limit solutions is the closure of the set of integral solutions when $F(t, \cdot)$ is full Perron or $A$ generates a compact semigroup. In the last case every control problem admits an optimal limit solution. We extend the existence and relaxation results of $[4,5,15,16]$.

(c) The existence of limit solutions can be also shown for a large class of evolution inclusions.

It appears that the notion of limit solutions is meaningful and it deserves further investigations.

Author Contributions: Conceptualization, methodology, investigation, writing-original draft preparation, writing-review and editing, T.D., S.B., O.C., N.J. and A.I.L. All authors contributed equally in writing this article. All authors have read and agreed to the published version of the manuscript.

Funding: The work of A. I. Lazu was supported by a grant of Romanian Ministry of Research and Innovation, CNCS - UEFISCDI, project number PN-III-P1-1.1-TE-2016-0868, within PNCDI III. The work of the other authors was supported by the Bulgarian National Science Fund under Project KP-06-N32/7.

Acknowledgments: The authors thank the reviewers for their valuable comments and suggestions which improved the paper.

Conflicts of Interest: The authors declare no conflict of interest.

\section{References}

1. Barbu, V. Nonlinear Differential Equations of Monotone Types in Banach Spaces; Springer: New York, NY, USA, 2010.

2. Ito, K.; Kappel, F. Evolution Equations and Approximations; World Scientific: Singapore, 2002.

3. Roubicek, T. Nonlinear Partial Differential Equations with Applications; Birkhauser: Basel, Switzerland, 2005.

4. Bothe, D. Nonlinear Evolutions in Banach Spaces; Habilitationsschritt: Paderborn, Germany, 1999.

5. Hu, S.; Papageorgiou, N. Handbook of Multivalued Analysis Volume II Applications; Kluwer: Dordrecht, The Netherlands, 2000.

6. Lakshmikantham, V.; Leela, S. Nonlinear Differential Equations in Abstract Spaces; Pergamon Press: Oxford, UK, 1981.

7. Benilan, P. Solutions intégrales d'équations d'évolution dans un espace de Banach. C. R. Acad. Sci. Paris Ser. 1972, A-B 274, A47-A50.

8. Cârjă, O.; Donchev, T.; Lazu, A.I. Generalized solutions of semilinear evolution inclusions. SIAM J. Optim. 2016, 26, 891-1409. [CrossRef]

9. Ważewski, T. On an optimal control problem. In Differential Equations and Their Applications; Springer: New York, NY, USA, 1963; pp. 229-242.

10. Bothe, D. Multivalued perturbations of m-accretive differential inclusions. Israel J. Math. 1998, 108, 109-138. [CrossRef]

11. Augustynowicz, A. Some remarks on comparison functions. Ann. Polon. Math. 2009, 96, 97-106. [CrossRef]

12. Tolstonogov, A. Differential Inclusions in a Banach Space; Springer: New York, NY, USA, 2000.

13. Bilal, S.; Cârjă, O.; Donchev, T.; Lazu, A.I. Nonlocal problems for evolution inclusions with one-sided Perron nonlinearities. RACSAM 2019, 113, 1917-1933. [CrossRef]

14. Donchev, T.; Farkhi, E. On the theorem of Filippov-Pliss and some applications. Control Cybernet. 2009, 38, 1251-1271.

15. Cârjă, O.; Donchev, T.; Postolache, V. Nonlinear evolution inclusions with one-sided Perron right-hand side. J. Dyn. Control Syst. 2013, 19, 439-456. [CrossRef]

16. Cârjă, O.; Donchev, T.; Postolache, V. Relaxation results for nonlinear evolution inclusions with one-sided Perron right-hand side. Set-Valued Var. Anal. 2014, 22, 657-671. [CrossRef] 
17. Migórski, S. Existence and relaxation results for nonlinear evolution inclusions revisited. J. Appl. Math. Stoch. Anal. 1995, 8, 143-149. [CrossRef]

18. Burlică, M.; Necula, M.; Roşu, D.; Vrabie, I.I. Delay Differential Evolutions Subjected to Nonlocal Initial Conditions; Monographs and Research Notes in Mathematics; CRC Press: New York, NY, USA, 2016.

19. Avgerinos, E.; Papageorgiou, N. Nonconvex perturbations of evolution equations with m-dissipative operators in Banach spaces. Comment. Math. Univ. Carolin. 1989, 30, 657-664.

20. Ahmed, R.; Donchev, T.; Lazu, A.I. Nonlocal m-dissipative evolution inclusions in general Banach spaces. Mediterr. J. Math. 2017, 14, 215. [CrossRef]

21. Vrabie, I. Compactness Methods for Nonlinear Evolutions; Wiley: Harlow, UK, 1995.

22. Ke, T.D. Cauchy problems for functional evolution inclusions involving $\mathrm{m}$-accretive operators. Electron. J. Qual. Theory Differ. Equ. 2013, 75, 1-13. [CrossRef]

23. Donchev, T. Functional differential inclusion with monotone right-hand side. Nonlinear Anal. 1991, 16, 533-542. [CrossRef]

(C) 2020 by the authors. Licensee MDPI, Basel, Switzerland. This article is an open access article distributed under the terms and conditions of the Creative Commons Attribution (CC BY) license (http:/ / creativecommons.org/licenses/by/4.0/). 